\title{
Detection of arrhythmias: use of a patient-activated ambulatory electrocardiogram device with a solid-state memory loop
}

\author{
A P BROWN, K D DAWKINS, J G DAVIES \\ From the Regional Cardiothoracic Unit, St George's Hospital, London
}

SUMMARY A patient-activated ambulatory electrocardiogram device with a solid-state memory loop was used to investigate 100 unselected patients experiencing palpitation, dizziness, or syncope. Clinically useful information was obtained in $68 \%$ of patients. Frequently, sinus rhythm had been restored by the time the patient activated the device, but the arrhythmia had been stored in the pre-event segment of the memory-a distinct advantage over many other patient-activated devices.

This recorder is complementary to conventional 24 hour electrocardiographic monitoring in the investigation of possible arrhythmias and is particularly suitable for patients with infrequent symptoms. Because the stored data can be rapidly analysed by any commercially available electrocardiograph the device is suitable for use in district general hospitals.

The role of ambulatory electrocardiographic monitoring is well established as a means of investigating cardiac rhythm disturbances. ${ }^{12}$ Despite this, the assessment of patients who present with palpitation, dizziness, or syncope may be difficult. In some the cause of the symptoms is non-cardiac; in others in whom the symptoms are related to an arrhythmia the disturbance of rhythm may be short lived or infrequent and a single 24 hour ambulatory electrocardiogram may fail to detect the arrhythmia or fail to correlate an arrhythmia with the symptoms. ${ }^{3}$

Another approach to the problem has been the use of portable recorders that can be activated when the patient has symptoms. ${ }^{4}$ These devices give no record of the rhythm preceding the acute event and arrhythmias lasting only a few seconds may not be detected at all. ${ }^{5}$ As an alternative, we describe the use of a reliable and inexpensive patient-activated device that has a pre-event solid-state memory facility.

\section{Patients and methods}

The recorder, designed by one of us (JGD), weighs $320 \mathrm{~g}$ (with batteries) and measures $146 \times 91 \times 34 \mathrm{~mm}$. It has no mechanical parts and

Requests for reprints to Dr K D Dawkins, Wessex Cardiothoracic Centre, Southampton General Hospital, Southampton SO9 4XY.

Accepted for publication 28 April 1987 uses a microprocessor to record a single channel electrocardiogram in a continuous 80 second loop. When the event button is pressed the loop stops and the data are stored in the solid state memory. The loop includes 70 seconds of rhythm before and $10 \mathrm{sec}-$ onds after the button is pressed. When convenient (up to six months after recording) the stored data are printed as rhythm strips by connecting the recorder to any ordinary electrocardiograph.

We reviewed the results of 106 consecutive and unselected patients who had used the recorder over a three year period. The case notes from six patients were incomplete; we studied the remaining 100 patients (aged 16-88 years (median 58 years)). Twenty six patients complained of palpitation alone, 38 had experienced presyncope, and 36 syncope with or without palpitation. Symptoms occurred at least once per week in 53 patients, at least once per month in a further 35 patients, and less than once a month in the remaining 12 patients. Table 1 shows their previous histories.

Forty two patients had already had conventional 24 hour electrocardiographic tape monitoring and 17 $(40 \%)$ had abnormal results. In these cases the solid state recorder was being used either because symptoms continued despite a change in treatment or because it was unclear whether the documented arrhythmias were the cause of the symptoms.

Each patient was fitted with the recorder for up to 
Table 1 Previous medical history

\begin{tabular}{lc}
\hline & No \\
\hline No known cardiac disease & 61 \\
Ischaemic heart disease & 23 \\
Valvar heart disease: & 5 \\
Mitral valve prolapse & 1 \\
Rheumatic mitral regurgitation & 2 \\
Aortic regurgitation & 1 \\
Aortic valve replacement & 2 \\
Atrial septal defect & 2 \\
Hypertrophic cardiomyopathy & 2 \\
Wolff-Parkinson-White syndrome & 1 \\
Dystrophia myotonica & $(100)$ \\
(Total) & \\
\hline
\end{tabular}

Table 2 Rhythm detected

\begin{tabular}{lr}
\hline & $N o$ \\
\hline Paroxysmal tachycardia: & 10 \\
$\quad$ Atrial fibrillation & 3 \\
Supraventricular tachycardia & 1 \\
Multiform ventricular tachycardia & \\
Frequent extrasystoles (1 in 5 or more): & 3 \\
Atrial & 9 \\
Ventricular & 27 \\
Occasional extrasystoles (atrial or ventricular) & 10 \\
Sinus tachycardia ( $>120$ beats/min) & \\
Bradycardia: & 1 \\
Junctional rhythm (rate 28 beats/min) & 1 \\
Sinus bradycardia (rate 25 beats/min) & \\
Sinus bradycardia and sinus arrest $(>13$ s) & 1 \\
Sinus arrest (2 s) & 1 \\
Intermittent atrioventricular dissociation & 2 \\
No events & 31 \\
(Total) & $(100)$ \\
\hline
\end{tabular}

*This patient had an episode of loss of consciousness and her husband pressed the event button. Because there was sinus bradycardia at the start of the recording the rhythm at the time of the acute event was never documented. She was paced and remains symptom free.

three weeks. The patient first returned to the electrocardiography department after recording two events or after one week. If no events had been recorded or if the rhythm was found to be sinus rhythm, or sinus rhythm with atrial or ventricular extrasystoles, new electrodes were applied and the patient was encouraged to continue recording for the remainder of the three week period.

\section{Results}

Of the 100 patients who used the recorder, 36 recorded one event and 33 made two or more recordings (table 2). For each patient only the most significant arrhythmia is shown. Fifty six patients made recordings that were considered completely diagnostic and these included 19 important arrhythmias. Thirteen further patients made recordings that were of some diagnostic value.

Fourteen episodes of paroxysmal tachycardia were recorded; multiform ventricular tachycardia was recorded in one patient (figure) and episodes of supraventricular tachycardia were recorded in the remainder. In five cases the arrhythmia was present at the start of the recording and the initiating event was not seen. Five of the patients with supraventricular tachycardia also had recordings showing sinus rhythm with infrequent atrial extrasystoles. In five of 14 cases of tachycardia and one of three cases of bradycardia the arrhythmia was only present in the pre-event segment of the memory (that is the patient had returned to sinus rhythm by the time the event button was pressed). All five patients with bradycardia or intermittent atrioventricular dissociation had suffered from syncope at some time; they therefore had a permanent endocardial pacemaker inserted and they remain symptom free.

Thirteen of the 19 patients with important arrhythmias had no previous cardiac history. Eight of the 12 patients with frequent extrasystoles had identical recordings on at least two occasions. The finding of normal sinus rhythm or occasional extrasystoles was regarded as diagnostic only if a similar recording was produced on two or more occasions in association with symptoms (14 of 27 patients). Thirty-one patients recorded no events; however, 10 of them had not used the recorder for the full three week period. Twelve of the patients with no events; had had infrequent symptoms ( $<1$ per month), and in seven drug treatment had been changed before they started to use the device.

Some patients found the recorder inconvenient to wear for long periods; other problems included loosening of the electrodes and patient interference with the recorder (for example removal of the batteries after recording had been made erased the memory). In the current model removal of the batteries does not erase the stored results which are backed up independently.

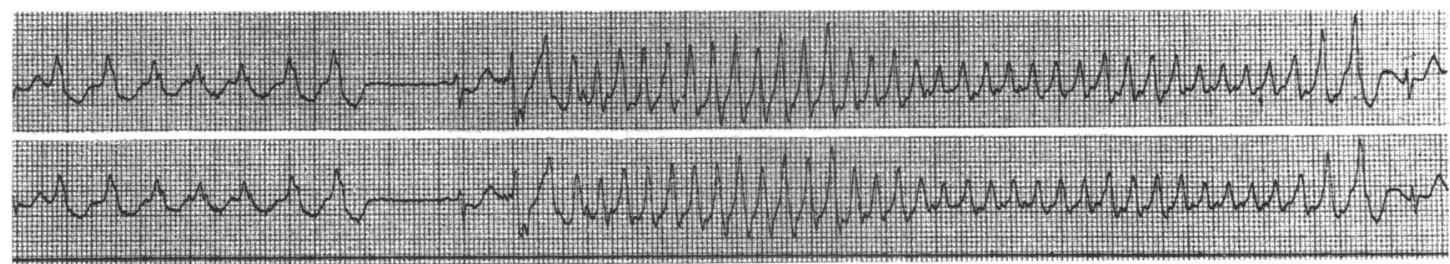

Figure Solid state recording of an episode of multiform ventricular tachycardia. 
Table 3 Comparison between 24 hour electrocardiographic tape and solid-state recorder

\begin{tabular}{lllll}
\hline \multicolumn{5}{c}{ Solid-state recorder } \\
\cline { 2 - 5 } & $\begin{array}{l}\text { Sinus rhythm } \\
\text { or extrasystoles }\end{array}$ & $\begin{array}{l}\text { Definite } \\
\text { abnormality }\end{array}$ & $\begin{array}{l}\text { No } \\
\text { events }\end{array}$ & Total \\
\hline 24 ECG tape & & & \\
$\begin{array}{c}\text { Sinus rhythm } \\
\text { or extra- } \\
\text { systoles }\end{array}$ & 11 & 6 & 8 & 25 \\
$\begin{array}{c}\text { Definite } \\
\text { abnormality }\end{array}$ & 5 & 7 & 2 & 14 \\
\hline
\end{tabular}

*We excluded three patients in whom drug treatment was

changed between the two investigations.

Table 3 compares the results of recordings obtained with the solid-state device and a conventional 24 hour electrocardiogram.

\section{Discussion}

These results show that this solid-state recorder may be of considerable diagnostic value in the assessment of patients with palpitation, dizziness, or syncope. Among our patients, of whom two thirds gave no previous history of cardiac disease, important arrhythmias were demonstrated in $19 \%$ and a further $22 \%$ had multiple extrasystoles or sinus tachycardia. The "negative" finding of normal sinus rhythm or occasional extrasystoles in $27 \%$ is also of value because the patient could be reassured that no important rhythm disturbance coincided with the symptoms. These data compare favourably with conventional 24 hour electrocardiographic monitoring, which can be expected to provide useful diagnostic information in up to $65 \%$ of recordings. ${ }^{6}$

The pre-event segment of memory is useful in several ways. Firstly, the initiating sequence of a rhythm disturbance can be documented; this may be crucial to the accurate identification of an arrhythmia. Second, short lived arrhythmias may be documented; in this study six of 17 patients with paroxysmal arrhythmias had returned to sinus rhythm by the time the event button was pressed. In this subgroup a diagnosis would not have been made by conventional patient-activated devices which only record from the moment the button is pressed. This factor may account for the poor results obtained with the Cardiomemo recorder, a patientactivated device without a pre-event memory. ${ }^{7}$

In six patients 70 seconds of pre-event memory were insufficient to capture the onset of the arrhythmia. In our current model the timing sequence may be varied to enable one recording of 160 seconds to be made, two of 80 seconds, four of $\mathbf{4 0}$ seconds, or eight of 20 seconds. A single 160 second recording is particularly appropriate for the patient with syncopal attacks as it may take one or two minutes for the patient to recover consciousness and activate the event button.

We were surprised that we only documented a single episode of ventricular tachycardia because a preponderance of atrial arrhythmias was noted with other patient-activated devices. ${ }^{48}$ This may merely reflect the relative frequency of supraventricular arrhythmias in a normal population ${ }^{6}$; an alternative explanation may be the small proportion $(23 \%)$ of patients with ischaemic heart disease in this study.

Because the recorder will only provide a record of symptomatic arrhythmias it is not a substitute for traditional 24 hour electrocardiographic monitoring in circumstances where asymptomatic arrhythmias are likely (such as ischaemic heart disease and dilated or hypertrophic cardiomyopathy). The use of the recorder is therefore complementary to 24 hour tape monitoring rather than a substitute for it (table 3). Future modifications of the microprocessor program to facilitate automatic recordings at times of asymptomatic arrhythmias would be an advantage. Early experience with a commercially available instrument, the Instant Replay transtelephonic monitor (Instromedix Inc), ${ }^{9}$ which includes many of the features of the device described in this paper, accords with our view of the usefulness of such recorders.

The simplicity of the recorder and the ease with which stored data can be analysed on standard equipment make the device suitable for use in district general hospitals.

\section{References}

1 McLeod AA, Jewitt DE. Role of 24-hour ambulatory electrocardiographic monitoring in a general hospital. Br Med J 1978;i:1197-9.

2 Winkle RA. Current status of ambulatory electrocardiography. Am J Cardiol 1981;102:757-70.

3 Petch MC. Lessons from ambulatory electrocardiography. Br Med J 1985;291:617-8.

4 Judson P, Holmes DR, Baker WP. Evaluation of outpatient arrhythmias utilizing transtelephonic monitoring. Am Heart J 1979;97:759-61.

5 Ward DE, Camm AJ. Ambulatory monitoring of transient arrhythmias. Hospital Update 1981;7:211-20.

6 Camm AJ, Ward DE, Spurrell RAJ. Arrhythmias in ambulatory persons: a review and experience of 1000 consecutive recordings. Biotelemetry 1978;5:167-81.

7 Thomas LE, Shapiro LM, Perrins EJ, Fox KM. Detection of arrhythmia: limited usefulness of patient activated recording devices. $\mathrm{Br} \mathrm{Med} J$ 1984;289: 1106-7.

8 Hasin Y, David D, Rogel S. Diagnostic and therapeutic assessment by telephone electrocardiographic monitoring of ambulatory patients. $\mathrm{Br}$ Med J 1976;ii: 609-12.

9 Hardwin Mead R, Ruder MA, Winkle RA. The "Instant Replay" transtelephonic monitor: utility in the diagnosis of intermittent symptomatic arrhythmias. PACE 1986;9:311. 